\title{
Retear rates after rotator cuff surgery: a systematic review and meta-analysis
}

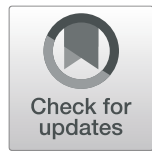

Umile Giuseppe Longo ${ }^{1 *} \mathbb{D}$, Arianna Carnevale ${ }^{1,2}$, llaria Piergentili ${ }^{1}$, Alessandra Berton ${ }^{1}$, Vincenzo Candela ${ }^{1}$, Emiliano Schena ${ }^{2}$ and Vincenzo Denaro ${ }^{1}$

\begin{abstract}
Background: Rotator cuff retear (RCR) is one of the main postoperative drawbacks. RCR can be considered a multifactorial issue, which causes are related either to biological than biomechanical factors. The aim of this study was to define the incidence of RCR after surgical treatment at different time points and to identify the main factors influencing the postoperative rotator cuff (RC) healing.

Methods: A systematic review and meta-analysis were performed following the PRISMA guidelines. A comprehensive search of the literature was carried out in July 2020, using PubMed and Cochrane Library databases. Only level 1 and 2 clinical evidence studies were included. Studies were included if patients with preoperative repairable full-thickness RC tears were treated surgically, and if studies reported postoperative RCR confirmed by imaging diagnostic. The association between timing of retear and follow-up time points were investigated using an inverse-variance method of pooling data. A subgroup meta-analysis was performed using the DerSimonian and Laird method for the estimation of the between-study variance, i.e., $\tau^{2}$. The association between retear rate after surgery and patients' age, preoperative tear size, fatty infiltration, postoperative rehabilitation protocol, surgical techniques, and RC repairs was determined by expressing the effect measure in terms of odds ratio (OR) with $95 \%$ confidence interval (Cl). The Mantel-Haenszel method with 95\% Cls was used.
\end{abstract}

Results: Thirty-one articles were included in this study. The percentage of RCR after surgery was $15 \%$ at 3 months follow-up, 21\% at 3-6 months follow-up, 16\% at 6-12 months follow-up, 21\% at 12-24 months follow-up, 16\% at follow-up longer than 24 months. The main factors influencing RC healing are both patient-related (i.e., age, larger tear size, fatty infiltration) and not patient-related (i.e., postoperative rehabilitation protocol, surgical techniques, and procedures).

Conclusions: Postoperative RC healing is influenced by patient-related and non-patient-related factors. Further high-level clinical studies are needed to provide highly relevant clinical results.

Keywords: Rotator cuff, Rotator cuff tear, Rotator cuff retear, Risk factors, Timing of retear

\footnotetext{
* Correspondence: g.longo@unicampus.it

'Department of Orthopaedic and Trauma Surgery, Campus Bio-Medico

University, Via Álvaro del Portillo, 200, Trigoria, 00128 Rome, Italy

Full list of author information is available at the end of the article
}

(c) The Author(s). 2021 Open Access This article is licensed under a Creative Commons Attribution 4.0 International License, which permits use, sharing, adaptation, distribution and reproduction in any medium or format, as long as you give appropriate credit to the original author(s) and the source, provide a link to the Creative Commons licence, and indicate if changes were made. The images or other third party material in this article are included in the article's Creative Commons licence, unless indicated otherwise in a credit line to the material. If material is not included in the article's Creative Commons licence and your intended use is not permitted by statutory regulation or exceeds the permitted use, you will need to obtain permission directly from the copyright holder. To view a copy of this licence, visit http://creativecommons.org/licenses/by/4.0/ The Creative Commons Public Domain Dedication waiver (http://creativecommons.org/publicdomain/zero/1.0/) applies to the data made available in this article, unless otherwise stated in a credit line to the data. 


\section{Background}

Rotator cuff (RC) tears are one of the leading causes of shoulder pain [1]. RC repair aims to re-attach the injured tendon in its native location. Despite the increase in the number of $\mathrm{RC}$ repair [2,3], rotator cuff retear (RCR) is one of the main postoperative drawbacks.

RCR can be considered a multifactorial issue, which causes are related either to biological than biomechanical factors. The first ones concern patient-related preoperative characteristics, such as age and tear size. RC retear rate increases with patients' age $[4,5]$. Also, the preoperative tear size has been reported as a factor that negatively influences the $\mathrm{RC}$ healing after surgery, as the size increases [5-7]. Other factors that have been related to higher healing failure include diabetes, smoking, preoperative fatty infiltration, and muscle atrophy [8-11]. The biomechanical characteristics of the repaired tendons may also be affected by surgical procedures (e.g., arthroscopic, open, mini-open repairs) and fixation techniques (e.g., single-row, double-row, suture bridge, transosseous repairs) [12-14]. Despite the advantages brought by the evolution of surgical techniques, the debate on which implies a lower retear rate after surgery still remains open [1416]. Timing of retear may be affected by the postoperative rehabilitation protocols, and structural integrity investigation at different time points of follow-up is controversial $[1,17-19]$. Some studies showed that retear is most frequent within 3 to 6 months postoperatively [20, 21]. During this postoperative period, no unanimous agreement exists about the best timing and strategy of rehabilitation protocol [22-24].

RCR rate after surgical repair ranges from 11 to $94 \%$ [25]. This high variability of RCR raises several concerns about the standards of medical care for $\mathrm{RC}$ diseases and adversely affect patients' expectations. The highest retear rate could be explained by an inadequate selection of patients, or by the inappropriate surgical procedures. The lowest retear rate may suggest both inadequate diagnostic imaging and follow-up time points to quantify more accurately structural integrity of RC after surgical repair. Furthermore, the timing of retears is not well-defined. To identify the most critical moment in which the probability of retears is higher could suggest modification of activities after surgery, or the application of biological solutions to improve RC healing. Moreover, improving the capability to identify patients at higher risk of retears could suggest alternative treatment options and impact positively on clinicians' decision making.

Therefore, this meta-analysis aimed to define the incidence of RCR after surgical treatment at different time points. Secondly, this investigation aimed to identify the main factors influencing the postoperative $\mathrm{RC}$ healing, with emphasis on preoperative patients' features, surgical procedures, and postoperative rehabilitation protocol.

\section{Methods}

\section{Search strategy}

A systematic review and meta-analysis were performed following the Preferred Reporting Items for Systematic Review and Meta-Analyses (PRISMA) guidelines [26]. A comprehensive search of the literature was performed from inception through July 2020, in PubMed and Cochrane Library databases. The search was conducted separately by two authors (IP, AC). The following search terms were used: ("Rotator Cuff Injuries" OR "Rotator Cuff Tear" OR "Rotator Cuff Retear") AND ("risk factor" OR age OR "tear size" OR diabetes OR smoking) AND ("timing of retear" OR "magnetic resonance imaging” OR MRI OR Arthro-MRI OR ultrasound).

\section{Criteria for including studies Types of studies}

Level 1 and 2 clinical evidence studies, as defined by the Oxford Centre for Evidence-Based Medicine, were included [27]. Nonrandomized studies, retrospective studies, case series, systematic reviews, and meta-analysis were not included. Only full-length English-language articles were considered for inclusion.

\section{Types of participants}

Studies were considered eligible for inclusion if they enrolled patients with repairable full-thickness RC tears, as confirmed preoperatively by imaging diagnostic (e.g., magnetic resonance imaging - MRI, ultrasound - US, computed tomography - CT). Partial RC tears were excluded. No limitations have been placed for inclusion about the preintervention $\mathrm{RC}$ tears size, namely small, medium, large, and massive tears have been included.

\section{Types of interventions}

Studies were included if RC tears were treated surgically, and if they reported postoperative rehabilitation protocols, including immobilization period, passive and active range of motion (ROM), strengthening exercises.

\section{Outcome measures}

Studies were eligible for inclusion if RCR was reported as an outcome measure, confirmed by postoperative imaging diagnostic. Only full-thickness defects have been counted for the postoperative retear rate. For those studies reporting postoperative tear according to the classification of Sugaya (types I-V) [28], only types IV and V were considered as representing full-thickness tears.

\section{Data collection and analysis \\ Studies selection process}

After duplicates removal, two reviewers (IP, AC) performed the titles and abstracts screening independently. The subsequent screening of the full-texts was carried 
out separately by the same two reviewers to examine in detail if studies met the inclusion criteria; in case of disagreement, a third reviewer (UGL) made the final decision. The reference list of the included studies was manually screened to retrieve additional studies, not resulting in the first search.

\section{Data extraction}

Data extraction was performed independently by two authors (IP, AC) using a predetermined checklist. The following data were extracted from studies that met the inclusion criteria: first author and year of publication, study design and level of evidence, randomization groups, basic patients demographic information (i.e., age, gender), postoperative rehabilitation protocol, i.e., immobilization (Yes/No) and correspondent duration (week), beginning of passive ROM (day), active assisted ROM (mean week), full active ROM (mean weeks), strengthening exercises (mean weeks), surgical technique (i.e., arthroscopy, open, mini-open), preoperative tears size according to Cofield classification as small $(<1 \mathrm{~cm})$, medium $(1-3 \mathrm{~cm})$, large $(3-5 \mathrm{~cm})$, massive $(>5 \mathrm{~cm})$ [29], $\mathrm{RC}$ repair (i.e., single-row, double-row, suture bridge, transosseous), diagnostic imaging tools (i.e., MRI, US, $\mathrm{CT}$ ), number of patients undergoing postoperative diagnostic imaging and follow-up (mean months), number of retears either in each single randomization group than overall and correspondent retear rate, fatty infiltration of cuff muscles before surgery.

\section{Risk-of-Bias assessment}

All studies that met the inclusion criteria were assessed for risk of bias by two authors independently (AC, VC). In case of disagreement, a third author (UGL) has been summoned to reach the final consensus. The internal validity criteria list proposed by van Tulder et al. has been considered for assessing the risk of bias [30]. The latter has been modified according to the guidelines from Cochrane Handbook for Systematic Reviews of Interventions [31]. The final list included 15 criteria for assessing selection bias, performance bias, attrition bias, detection bias, and reporting bias. The included domains concerned sequence generation, allocation concealment, blinding of participants, selective outcome reporting, incomplete data addressed, personnel, and outcome assessors. This methodological quality assessment is in accordance with the PRISMA protocol [32]. Each criterion was evaluated, assigning a score of 0 for low risk, 1 for uncertain, and 2 for high risk of bias. Thus, the total score range for quality assessment was 0-30: a high score implied a lower quality level, and a low score implied a higher quality level. The quality level of the included studies was evaluated as high if total score $\leq 5$, moderate if $5<$ total score $\leq 7$, and low if total score $>7$.

\section{Data analysis and statistical methods}

The statistical analysis was performed using $\mathrm{R}$ software version i368 3.6.1. The retear rate was calculated as the number of patients reporting a not healed tendon after surgery to the total number of patients undergoing RC surgery. Tendons health evaluations refer to imaging examinations performed at the same follow-up time point.

The association between timing of retear and followup time points were investigated using an inversevariance method of pooling data. Pooled retear rate estimates at different time points were performed subdividing follow-up periods into different subgroups, i.e., within 3 months ( $\leq 3$ months), after 3 months within 6 months $(3<$ months $\leq 6)$, after 6 months within 12 months $(6<$ months $\leq 12)$, after 12 months within 24 months $(12<$ months $\leq 24)$, and after 24 months (months $>24$ ). The subgroups meta-analysis was performed using a random-effect model and the DerSimonian and Laird method for the estimation of the between-study variance, i.e., $\mathrm{T}^{2}$. The $\mathrm{I}^{2}$ statistic was applied to define if there was heterogeneity within results. The heterogeneity was interpreted according to the Cochrane Handbook for Systematic Reviews of Interventions guidelines [31]. A random-effects model has been applied to include heterogeneity among studies.

The association between retear rate after surgery and patients' age, preoperative tear size, fatty infiltration, postoperative rehabilitation protocol, surgical techniques, and RC repairs, was determined by expressing the effect measure in terms of odds ratio (OR) with $95 \%$ confidence interval (CI). The OR indicated the ratio of the probability that retear event occurred after RC surgery to the probability that it did not occur. The Mantel-Haenszel method with 95\% CIs was used.

\section{Results}

\section{Search finding}

The results of the literature search, screening, review, and inclusion in quantitative synthesis are reported in Fig. 1. The initial search yielded a total of 1397 articles, with additional 2 articles included after manual reference list screening. After duplicates removal, a total of 933 studies were screened based on title and abstract, of which 839 records were excluded because not relevant for our objective. A total of 94 articles were analyzed in detail, of which 35 records were excluded because they did not satisfy the inclusion criteria. A total of 59 studies were evaluated for methodological quality, 31 of which resulted eligible for meta-analysis [12, 13, 15, 28, 33-87]. None of the studies included the same population. For the complete table, including all extracted data, see Additional file 1 and Additional file 2. 


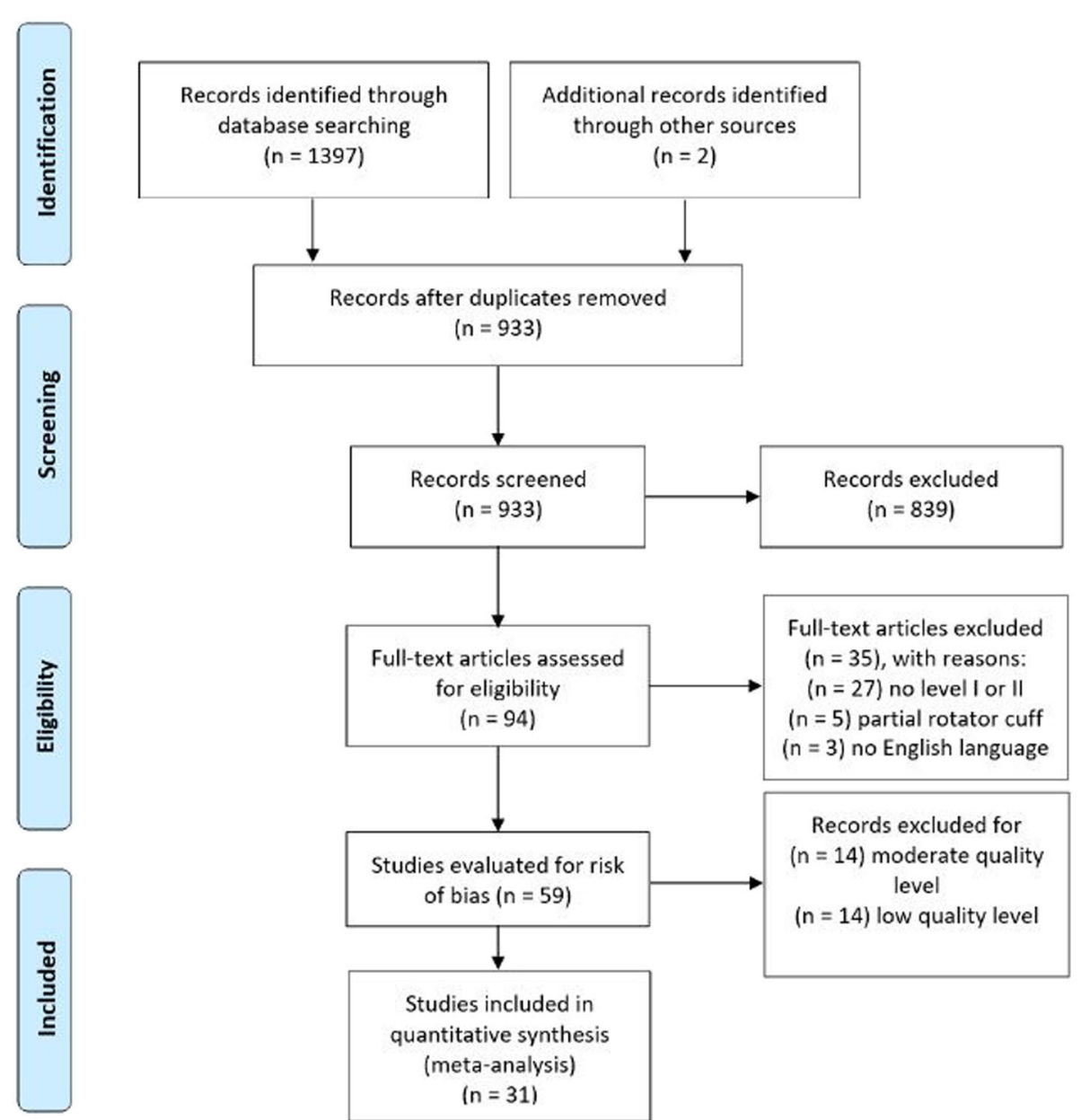

Fig. 1 PRISMA flow diagram for studies selection

\section{Risk of bias}

The included studies showed an average risk-of-bias score of 5.63 (range, 0-30) (Additional file 3). Of the 59 studies satisfying the inclusion criteria, 31 (52.54\%) studies showed a high-quality level, 14 (23.73\%) a moderatequality level, and $14(23,73 \%)$ a low-quality level.
Timing of retears at different follow-up time points

The elapsed time between the $\mathrm{RC}$ repair and follow-up of structural integrity examination by diagnostic imaging ranged from 1 month to 60 months (mean \pm standard deviation [SD], $13.7 \pm 11$ ). The percentage of RCR after surgery was $15 \%$ at 3 months follow-up (Fig. 2), 21\% at

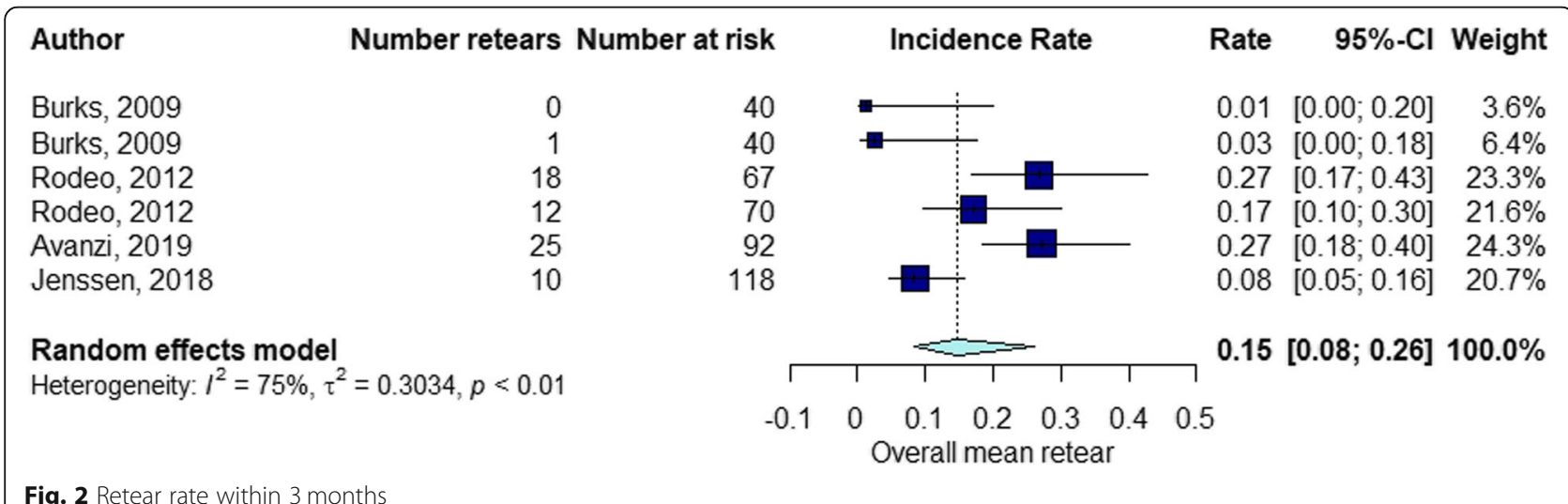


3-6 months follow-up (Fig. 3), 16\% at 6-12 months follow-up (Fig. 4), 21\% at 12-24 months follow-up (Fig. 5), 16\% at follow-up longer than 24 months (Fig. 6).

\section{Retear rate and patient-related risk factors Age}

A total of 31 studies were analysed to examine the relationship between retear rate and patients' age. The weighted average age of the included studies was 58.2 years $( \pm 3.7 \mathrm{SD})$, ranging from 53 to 67 years old. Splitting by different decades of age, studies with a mean age less than equal to 60 years and studies with a mean age over 60 years were compared. The retear rate for patients under 60 years of age was $14.4 \%$. The retear rate for patients over 60 years of age was $24.3 \%$. Older age is associated with higher retear rate (OR, 1.8; 95\% CI, 1.5 to $2.3 ; P<0.0001$ ) (Table 1 ).

\section{Tear size}

A total of 11 studies were analysed to examine the relationship between retear rate and tear size before surgery. Two subgroups were analysed: group A included only small, medium, or small-to-medium tears; group B included only large, massive, or large-to-massive tears. The average retear rate was $12.5 \%$ for group A and $37 \%$ for group B. Larger tears were associated with higher retear rate $(\mathrm{OR}, 0.3 ; 95 \% \mathrm{CI}, 0.2$ to $0.5 ; \mathrm{P}<0.0001)$ (Table 1).

\section{Fatty infiltration}

A total of 3 studies were analysed to examine the relationship between the retear rate and presurgical GFDI (global fatty degeneration index). The weighted average of GFDI of the included studies was $1.6( \pm 0.5 \mathrm{SD})$. Fatty degenerations greater than grade 1.43 correspond to a higher likelihood or retear recurrence [88]. Grade 1.43 of GFDI was used as threshold for discriminating between a high probability of tendon integrity and a high probability of retear. The retear rate was 15.4 and $14.6 \%$ for subgroups with GFDI lower than and higher than the threshold, respectively. Results showed no statistically significant difference (OR, $0.9 ; 95 \% \mathrm{CI}, 0.4$ to 1.9 ; $P=0.7588)$ (Table 1).

\section{Retear rate and postoperative rehabilitation protocol Immobilization period}

A total of 22 studies were analysed to examine the relationship between the retear rate and the immobilization period. According to the rehabilitation protocol of Multicenter Orthopaedic Outcomes Network Shoulder group (the MOON Shoulder Group) [89], the analysis was performed by comparing the retear rate for patients who wore the sling for up to 6 weeks (subgroups 1) to retear rate for patients who were immobilized for more than 6 weeks (subgroups 2). The average retear rate was 17.8 and $8.3 \%$ for the subgroups 1 and the subgroup 2, respectively. Results showed no statistically significant difference $(\mathrm{OR}, 0.4 ; 95 \% \mathrm{CI}, 0.1$ to $1.2 ; P=0.0912)$ (Table 1).

\section{Passive ROM}

A total of 27 studies were analysed to examine the relationship between the retear rate and the beginning of passive ROM. According to the MOON Shoulder Group, in the early group, passive ROM exercises start from 1 day to 1 week after surgery [89]. The analysis was performed by comparing the retear rate for patients who performed early passive ROM ( $\leq 7$ days after surgery) to retear rate for patients who performed delayed passive ROM ( $>7$ days after surgery). The average retear rate was $17.5 \%$ for the "early subgroup" and $15.6 \%$ for the "delayed subgroup". Results showed no statistically significant difference $(\mathrm{OR}, 0.8 ; 95 \% \mathrm{CI}, 0.7$ to $1.1 ; P=$ 0.1237) (Table 1).

\section{Active assisted ROM}

A total of 23 studies were analysed to examine the relationship between the retear rate and the beginning of active-assisted ROM. The weighted average of weeks of active-assisted ROM of the included studies was $6( \pm 2.6$

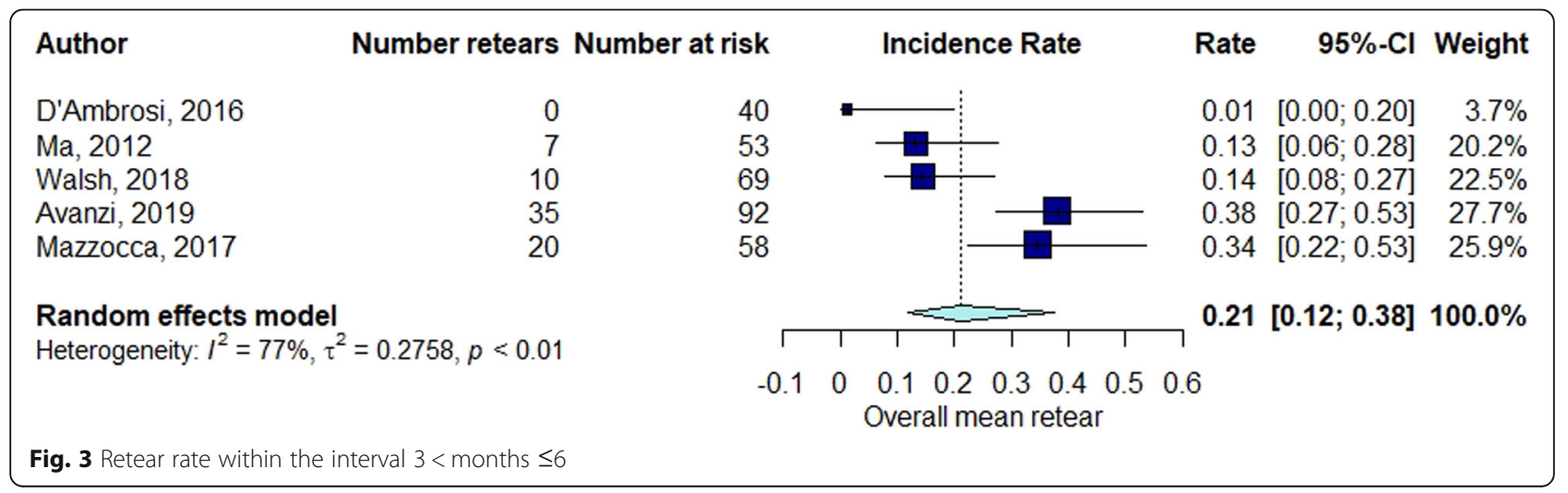




\begin{tabular}{|c|c|c|c|c|c|c|}
\hline Author & Number retears & Number at risk & Incidence Rate & Rate & $95 \%-\mathrm{Cl}$ & Weight \\
\hline Burks, 2009 & 3 & 40 & & 0.07 & {$[0.02 ; 0.23]$} & $4.0 \%$ \\
\hline Kim, 2016 & 14 & 82 & & 0.17 & {$[0.10 ; 0.29]$} & $7.3 \%$ \\
\hline Lapner, 2012 & 21 & 76 & & 0.28 & {$[0.18 ; 0.42]$} & $7.9 \%$ \\
\hline Malavolta, 2018 & 1 & 44 & $=$ & 0.02 & {$[0.00 ; 0.16]$} & $1.9 \%$ \\
\hline Zumstein, 2016 & 13 & 35 & & 0.37 & {$[0.22 ; 0.64]$} & $7.2 \%$ \\
\hline Avanzi, 2019 & 32 & 90 & & 0.36 & {$[0.25 ; 0.50]$} & $8.4 \%$ \\
\hline Jenssen, 2018 & 12 & 113 & & 0.11 & {$[0.06 ; 0.19]$} & $7.0 \%$ \\
\hline Koh, 2014 & 9 & 88 & & 0.10 & {$[0.05 ; 0.20]$} & $6.5 \%$ \\
\hline Sheps, 2019 & 52 & 165 & & 0.32 & {$[0.24 ; 0.41]$} & $8.7 \%$ \\
\hline Lee, 2016 & 20 & 128 & & 0.16 & {$[0.10 ; 0.24]$} & $7.8 \%$ \\
\hline Nam, 2018 & 6 & 41 & & 0.15 & {$[0.07 ; 0.33]$} & $5.6 \%$ \\
\hline Liu, 2017 & 9 & 99 & & 0.09 & {$[0.05 ; 0.17]$} & $6.5 \%$ \\
\hline van der Zwaal, 2013 & 14 & 95 & & 0.15 & {$[0.09 ; 0.25]$} & $7.3 \%$ \\
\hline Rhee, 2012 & 14 & 110 & & 0.13 & {$[0.08 ; 0.21]$} & $7.3 \%$ \\
\hline Keener, 2014 & 9 & 116 & & 0.08 & {$[0.04 ; 0.15]$} & $6.5 \%$ \\
\hline \multicolumn{4}{|c|}{ Random effects model } & \multirow{2}{*}{\multicolumn{2}{|c|}{$0.16[0.12 ; 0.22]$}} & $100.0 \%$ \\
\hline \multirow{2}{*}{\multicolumn{2}{|c|}{ Heterogeneity: $I^{2}=78 \%, \tau^{2}=0.2499, p<0.01$}} & 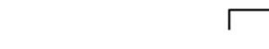 & 1 & & & \\
\hline & & -0.2 & $\begin{array}{llll}0 & 0.2 & 0.4 & 0.6\end{array}$ & 0.8 & & \\
\hline
\end{tabular}

SD). According to the MOON Shoulder Group, the early active-assisted ROM start before 5 weeks post-surgery and the delayed active-assisted ROM start after 5 weeks post-surgery [89]. Two subgroups were analyzed: the first group and the second group included patients who performed the active-assisted ROM before and after 5 weeks, respectively. The retear rate was $25.6 \%$ for the first group, and $14.2 \%$ for the second group. Starting active-assisted ROM before 5 weeks corresponds to a higher retear rate (OR, 0.5; 95\% CI, 0.4 to $0.7 ; P<0.0001$ ) (Table 1 ).

\section{Full active ROM}

A total of 9 studies were analysed to examine the relationship between the retear rate and the beginning of full active ROM. The weighted average of weeks of full active ROM of the included studies was $8.2( \pm 2.6 \mathrm{SD})$. According to the MOON Shoulder Group, two subgroups were analysed: the first group and the second group included patients who performed the full active ROM before and after 8 weeks, respectively [89]. The retear rate was $12.1 \%$ for the first group, and $21.8 \%$ for the second group. Starting full active ROM after 8 weeks corresponds to a higher retear rate (OR, 2; 95\% CI, 1.3 to $3.2 ; P=0.0028)$ (Table 1 ).

\section{Strengthening exercises}

A total of 23 studies were analysed to examine the relationship between the retear rate and the beginning of

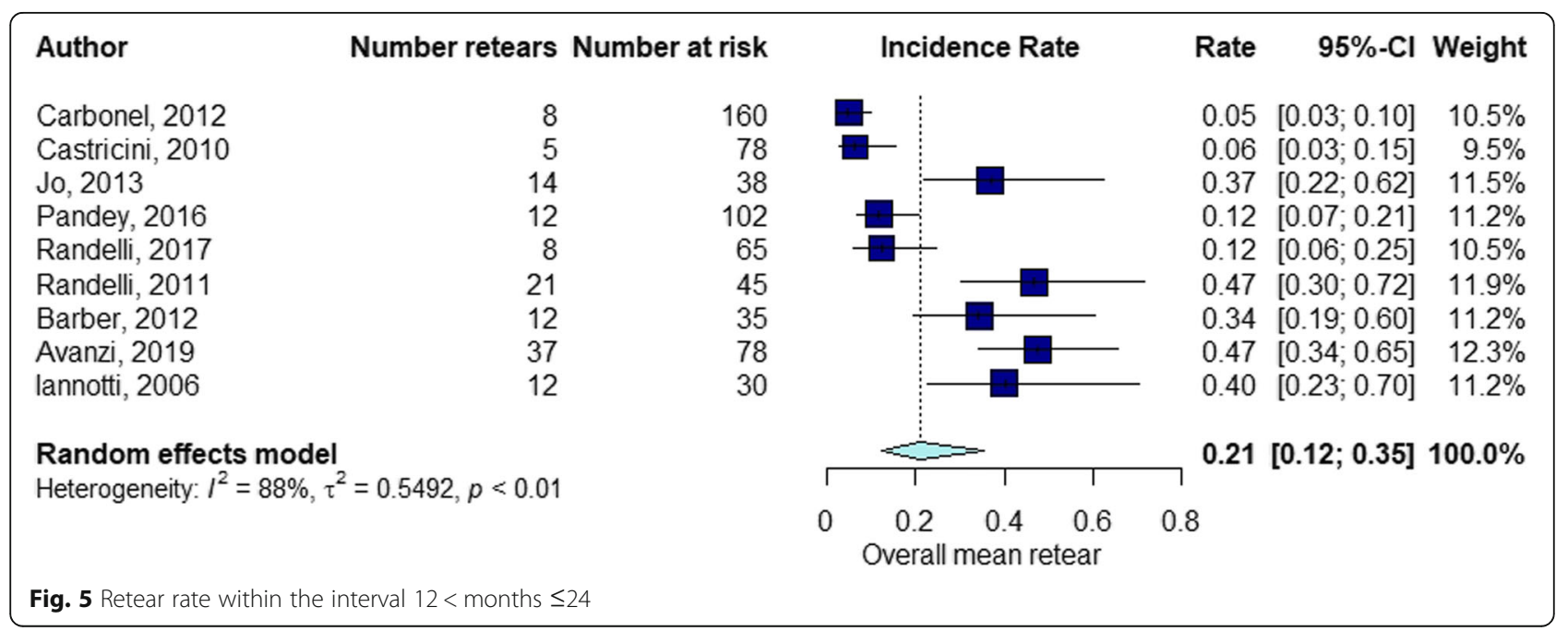




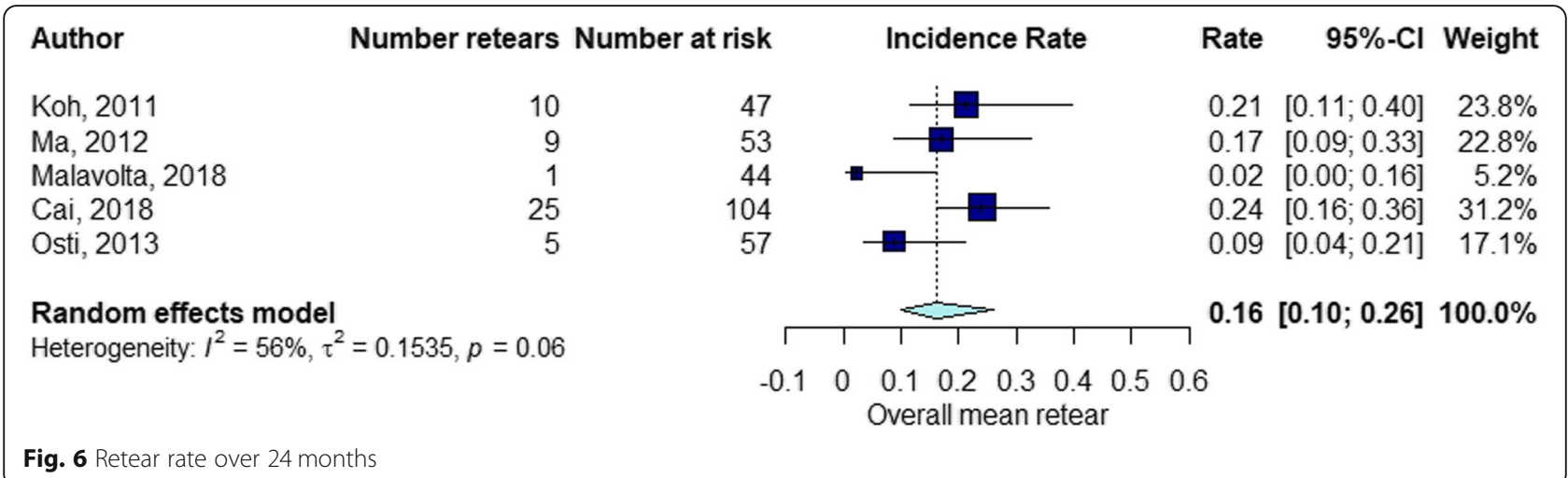

strengthening exercises. The weighted average of weeks of strengthening exercises of the included studies was 11.3 ( $+4 \mathrm{SD})$. According to the MOON Shoulder Group, strengthening exercises are usually recommended starting at the 12th week after surgery [89]. Two subgroups were analysed: the first group and the second group included patients who performed the strengthening exercises before and after 12 weeks, respectively. The retear rate was $14.5 \%$ for the first group, and $15.9 \%$ for the second group. Results showed no statistically significant difference (OR, 1.1; $95 \% \mathrm{CI}, 0.8$ to 1.5; $P=0.4653$ ) (Table 1).

\section{Retear rate and surgical techniques \\ Arthroscopic versus open/mini-open}

A total of 31 studies were analysed to examine the relationship between the retear rate and the performed surgical procedures. Two groups were analysed: the first group included patients who underwent arthroscopic surgery, and the second group included patients who underwent open and/or mini-open surgery. The average retear rate was $17.3 \%$ for the first group, and $21.8 \%$ for the second group. Results showed no statistically significant difference (OR, 1.0; 95\% CI, 0.7 to $1.7 ; P=0.8524)$ (Table 1).

\section{Single-row versus double-row}

A total of 18 studies were analysed to examine the relationship between retear rate and single-row or doublerow RCR. The average retear rate was $14.5 \%$ for patients who underwent single-row repair, and $12.7 \%$ for patients underwent double-row. Results showed no statistically significant difference (OR, 1.3; $95 \% \mathrm{CI}, 0.9$ to $1.9 ; P=$ 0.2036) (Table 1).

\section{Single-row versus suture bridge/transosseous}

A total of 22 studies were analysed to examine the relationship between retear rate and single-row or suture bridge/transosseous RC repair. The average retear rate was $14.5 \%$ for patients who underwent single-row repair,

Table 1 Comparison of risk factors for retear rate

\begin{tabular}{llll}
\hline Risk factors & No. of Studies & Odds ratio (95\% Cl) & $P_{\text {-value }}$ \\
\hline Age & 31 & $1.8(1.5-2.3)$ & $<0.0001^{*}$ \\
Tear size, A Vs. B & 11 & $0.3(0.2-0.5)$ & $<0.0001^{*}$ \\
Fatty infiltration (GFDI) & 3 & $0.9(0.4-1.9)$ & 0.7588 \\
Immobilization period (6 weeks) & 22 & $0.4(0.1-1.2)$ & 0.0912 \\
Passive range of motion (7 days) & 27 & $0.8(0.7-1.1)$ & 0.1237 \\
Active assisted ROM (5 weeks) & 23 & $0.5(0.4-0.7)$ & $<.0 .0001^{*}$ \\
Full active ROM (8 weeks) & 9 & $2.0(1.3-3.2)$ & $0.0028^{*}$ \\
Strengthening exercises (12 weeks) & 23 & $1.1(0.8-1.5)$ & 0.4653 \\
Arthroscopic vs. open/mini-open & 31 & $1.0(0.7-1.7)$ & 0.8524 \\
Single-row vs. double-row & 18 & $1.3(0.9-1.9)$ & 0.2036 \\
Single-row vs. suture bridge/transosseous & 22 & $0.6(0.4-0.8)$ & $0.0005^{*}$ \\
Double-row vs. suture bridge/transosseous & 15 & $0.5(0.3-0.7)$ & $0.0001^{*}$ \\
PRP vs. No PRP & 9 & $0.6(0.4-0.9)$ & $0.0179^{*}$ \\
Tendon augmentation vs. No augmentation & 4 & $0.2(0.1-0.4)$ & $<0.0001^{*}$ \\
\hline
\end{tabular}

${ }^{\mathrm{a}} \mathrm{A}=$ small, medium, small-to-medium tears, $\mathrm{B}=$ large, massive, large-to-massive tears 
and $23.6 \%$ for patients who underwent suture bridge/ transosseous RC repair. Suture bridge/transosseous repairs correspond to a higher retear rate than single-row procedure (OR, $0.6 ; 95 \% \mathrm{CI}, 0.4$ to $0.8 ; P=0.0005$ ) (Table 1).

\section{Double-row versus suture bridge/transosseous}

A total of 15 studies were analysed to examine the relationship between retear rate and double-row or suture bridge/transosseous RC repair. The average retear rate was $12.7 \%$ for patients who underwent double-row repair, and $23.6 \%$ for patients who underwent suture bridge/transosseous repair. Suture bridge/transosseous repairs correspond to a higher retear rate than doublerow procedure $(\mathrm{OR}, 0.5 ; 95 \% \mathrm{CI}, 0.3$ to $0.7 ; P=0.0001$ ) (Table 1).

\section{Platelet-rich plasma (PRP)}

A total of 9 studies were analysed to examine the relationship between the retear rate and the use of PRP. Two subgroups were identified: the first group and the second group referred to the use or not of PRP, respectively. The average retear rate was $14.5 \%$ for the first group, and $23.9 \%$ for the second group. The use of PRP corresponds to a lower retear rate (OR, 0.6; 95\% CI, 0.4 to $0.9 ; P=0.0179$ ) (Table 1 ).

\section{Tendon augmentation.}

A total of 4 studies were analysed to examine the relationship between retear rate and tendon augmentation for RC repair. Two subgroups were identified: the first group with augmentation and the second group without augmentation. The average retear rate was $21.2 \%$ for the first group, and $51.2 \%$ for the second group. Tendon augmentation corresponds to a lower retear rate (OR, $0.2 ; 95 \%$ CI, 0.1 to $0.4 ; P<0.0001$ ) (Table 1 ).

\section{Discussion}

This study aimed to investigate the retear rate after RC surgery at different time points, also evaluating both patient-related and not patient-related factors. The first ones concern preoperative patients' characteristics, such as age, tear size, and fatty infiltration. The second ones are related to postoperative rehabilitation protocol, or intraoperative choices of surgical procedures, and RC repairs techniques. The present meta-analysis, including only level 1 and 2 evidence studies, reports data on over $2500 \mathrm{RC}$ repairs.

\section{RCR at different time points}

The results of the present study suggest a difference in retear rate at different follow-up time points corresponding to diagnostic imaging assessments. After surgery, the percentage of RCR was $15 \%$ at 3 months follow-up, $21 \%$ at 3-6 months follow-up, $16 \%$ at 6-12 months follow- up, $21 \%$ at $12-24$ months follow-up, $16 \%$ at follow-up longer than 24 months. These findings would suggest more frequent time points for diagnostic imaging between 3 and 6 months and 12-24 months after RC surgery. Our initial focus was to investigate when the retears occur at different time points in relation to the preoperative tear size. A stratified analysis could not be performed because of the insufficient number of studies reporting the preoperative tear size at different followup groups. Despite this limitation, this study could provide significant insights about future studies investigating the retear rate after RC repair. Better knowledge about the timing of retear could be beneficial to define guidelines for surgical procedures and postoperative management.

\section{Retear rate and patient-related risk factors}

The advanced age of patients and larger tear sizes are predictors of RC retear, in agreement with previously published studies $[4,6,20,90]$. The negative influence of older age on the tendons healing process also depends on other concomitant factors age-related, such as lifestyle, bone mineral density, comorbidities. Some studies report that older age is not an independent predictor of RCR [5]. Larger tear size has been associated with a higher retear rate also in previous systematic reviews [2, 91]. The results of our investigation confirm this association, showing a strong statistical significance $(P<0.0001)$. However, in the attempt to include as many as possible studies in the quantitative analysis, we identified two macro groups. The first group included studies reporting postoperative retear rate for patients with small and/or medium tear size, and the second one included studies enrolling only patients with large and/ or massive tear size. For this reason, only 11 studies were analyzed to investigate the relationship between preoperative tear size and retear rate, excluding those studies that enrolled patients independently from the preoperative tear size. Even if $97 \%$ of the included studies reported data about patients' age and preoperative tear size, no one has provided a direct association regarding the number of patients who experienced a retear and their presurgical features. Moreover, the collected information was not enough to perform a stratified analysis, so the definition of the independent effect of both patients' age and preoperative tear size was not possible.

Current literature reports that RCs with higher muscle fatty infiltration have an increased likelihood of suffering RCR [92]. Our results showed no statistically significant difference $(P=0.7588)$. This deniable statement agrees with the results reported in a recent study [91]. A plausible explanation is that there is no robust scientific evidence. Moreover, studies reporting data on preoperative fatty infiltration did not provide postoperative variations 
in fatty degeneration and any correlation with RC retear. Although fatty infiltration has been considered as one of the main factors influencing tendons healing after surgery [20,93], further clinical investigations should be performed to corroborate its impact with exhaustive evidence.

As reported in previous studies, additional patientrelated factors that could negatively influence the healing process of the repaired tendons are smoking, diabetes, osteoporosis, hyperlipidemia $[5,6]$. In the present work, these risk factors were not analyzed, and further investigations are needed to provide more robust clinical evidence.

\section{Retear rate and not patient-related risk factors}

The biomechanics of the repaired tendons may also be affected by not patient-related factors. In the postoperative period, patients may experience limited functionalities of the affected arm and pain. In this regard, wide debates arise in the definitions of the best rehabilitation programs that should minimize the risk of healing failure and guarantee a successful return to activities of daily living $[1,94]$. In the current clinical practice, the postoperative management of patients' underwent RC repair can be slightly different among studies in terms of time points in which start specific movements and physical exercises. Based on the available literature, the postoperative rehabilitation protocol could be split into four main phases [95]. The first phase refers to the immediate postoperative period until the 6-week during which supervised passive ROM and active-assisted ROM are allowed; in the second phase (weeks 6-12), patients start to execute full-active ROM; in the third phase (months $3-4)$, stretching and strengthening exercises can be initiated and continued in the fourth phase (months 4-6) to completely restore full and pain-free active ROM and return as normally as possible to sports, activities of daily living and work. The timing of immobilization has been investigated in some recent randomized controlled trials $[65,70,77]$. Longer periods of immobilization may result in shoulder stiffness, which negatively influences the healing process after surgery [81]. Commonly, most patients are asked to wear an abduction pillow for 3 to 6 weeks, during which home postural exercises and assisted ROM during physical therapy are prescribed $[65,84]$. The recommended immobilization period may change based on the preoperative tear size. At the same time, the effect of early passive mobilization on the healing rate after RC surgery has been investigated [64, 68, 84]. Compared with the delayed rehabilitation protocol, the early mobilization aims to avoid the likelihood that adhesions would give rise to shoulder stiffness; conversely, delayed rehabilitation protocol seeks to preserve the tendon-to-bone integrity, avoiding retear. One study compared the retear rate in two groups immobilized for four or eight weeks, avoiding any type of passive or active ROM exercises [67]. At a mean of 6.8 months, MRI showed a retear rate of $12.5 \%$ for 4-weeks immobilization group and a retear rate of $8.3 \%$ for 8 weeks immobilization group. The same study proposed a subgroup analysis, including only patients without preoperative shoulder stiffness. Results showed that at 24months postoperatively, the 8-week immobilization group had a higher percentage of patients with stiffness [67]. Such findings suggest that the risk of shoulder stiffness might be avoided executing balanced and limited ROM in the first weeks after surgery. Our analysis showed no statistically significant differences for immobilization periods within 6 weeks or longer than 6 weeks postoperatively (OR, $0.4 ; 95 \% \mathrm{CI} ; 0.1$ to $1.2 ; P=$ $0.0912)$. Some recent meta-analysis investigated the outcomes of early versus delayed rehabilitation [18, 24]. These studies report that early motion protocol corresponds to an increase of ROM after RC repair, but also the risk of retear increases. Based on our findings that larger RC tear size may experience a lower healing rate, we suggest that early motion could be recommended for smaller tear size, while the delayed motion for larger tear size. In the first phase of rehabilitation (within the 5 weeks), active-assisted ROM should be avoided, since a higher retear rate was found for active-assisted ROM starting before 5 weeks (OR, $0.5 ; 95 \% \mathrm{CI}, 0.4$ to 0.7 ; $P<0.0001$ ). In the second phase of rehabilitation (weeks 6-12), full active ROM can be recommended; in particular, our results suggest a higher healing rate if full active ROMs are started before the 8th week (OR, 2; 95\% CI, 1.3 to $3.2 ; P=0.0028)$. Usually, strengthening exercises are recommended after the 12 th week $[28,57,85]$, when full active ROM and dynamic shoulder stabilization should be reached [95]. Our results suggest a higher healing rate if strengthening exercises are started before the 12th week, although this result showed no statistically significant difference (OR, 1.1; $95 \% \mathrm{CI}, 0.8$ to 1.5 ; $P=0.4653)$.

During these periods, patients' compliance with immobilization and prescribed movements should be analyzed $[65,96]$. Monitoring patients using wearable technologies could be a plausible alternative if compared to a questionnaire-based investigation [1, 97]. As highlighted previously, tendons healing is strictly associated with factors that surgeons could not handle totally because of dependence from patients' biological characteristics, as age, tear size, muscles fatty degeneration, and atrophy. Due to the heterogeneity of the included studies and insufficient available data, a stratified analysis for the determination of the independent effect of each factor was not possible to carry out. 
In the last decades, arthroscopic RC repair supplanted previous techniques thanks to progress in surgical and technological instrumentations [98]. Faster recovery and better cosmetic results have been the main reasons to prefer the arthroscopic approach. Further studies also supported good clinical outcomes and a low retear rate [99]. The present investigation did not show statistically significant results comparing the retear rate associated with arthroscopic or open and mini-open RC repair (OR, 1; 95\% CI, 0.7 to $1.7 ; P=0.8524$ ).

Our data indicated that double-row techniques yield a lower retear rate than suture bridge/transosseous (OR, 0.5 ; $95 \% \mathrm{CI}, 0.3$ to $0.7 ; P=0.0001$ ) and single-row $\mathrm{RC}$ repair, although there was no statistically significant difference for the latter (OR, 1.3; 95\% CI, 0.9 to $1.9 ; P=$ 0.2036). The present study showed that single-row RC repair is associated with a lower retear rate compared to suture bridge/transosseous RC repair (OR, 0.6; 95\% CI, 0.4 to $0.8 ; P=0.0005$ ).

Double-row RC repair has been described as biomechanically superior compared with single-row [100]. According to our study, numerous systematic reviews and meta-analyses have shown lower postoperative retear rate after double-row repair.

Our findings partially agree with those of Hein et al. that found that double-row had significantly lower retear rate compared with single-row [16]. However, they did not find any significant difference between double-row and suture bridge/transosseous and significantly lower retear rate with suture bridge/transosseous than singlerow. Possible reasons for dissimilar results could be patient population, follow-up time, methods for retear diagnosis, and sample size.

As this study focuses on the retear rate based on imaging-classification, no conclusions regarding clinical outcomes as a function of repair technique can be made. Yang et al. demonstrated that postoperative RCR alters clinical outcomes [101]. Future studies should compare differences in the effect based on repair types.

PRP is a promising treatment for some musculoskeletal diseases; however, evidence of its efficacy in the treatment of RC pathologies is still insufficient. Several studies focused on PRP injection for RC tendinopathy, showing benefits over sham injection, no injection, or physiotherapy alone in reducing pain at long-term follow-up [102]. In this review, we analyzed evidence of PRP in arthroscopic repair of RC tears compared with conventional surgery. Lower retear rate was observed with the use of PRP, but several aspects need to be further focused. Many of the included studies specifically looked at the use of platelet-rich fibrin matrix for augmentation (PRFM), while others injected PRP directly into the repair site, including leukocyte-rich PRP (LRPRP) and leukocyte-poor PRP (LP-PRP). The differences in concentration, content, preparation method and delivery technique do not allow to derive definitive conclusions. Moreover, patients were not stratified based on concomitant factors that can affect the retear rate, such as size, chronicity, atrophy, fatty infiltration, patients' age, use of tobacco products, diabetes, and other patient-related factors.

Previous studies confirm that PRP has effects on RC structural integrity, promoting tendon healing to the bone, but no effects on clinical outcomes were observed after RC repair. As mentioned above, the present review focused on the retear rate based on imagingclassification, and no conclusions regarding clinical outcomes as a function of repair technique can be made. The efficacy of PRP in arthroscopic repair of RC tears remains under investigation.

The results of this review show that augmented $\mathrm{RC}$ repair has a lower retear rate. Structural integrity in postoperative imaging has been documented, but literature is still insufficient.

\section{Conclusions}

Retear rate after RC surgical repair is found to be $15 \%$ within 3 months after surgery, $16 \%$ at $6-12$ months follow-up and at follow-up longer than 24 months, $21 \%$ at 3-6 months and 12-24 months follow-up. Advanced patients' age, larger tear size, and fatty infiltrations are factors influencing the RC healing negatively. Future high-level clinical studies should report data on patients' condition, postoperative rehabilitation protocol, and surgical techniques in a standardized way to perform a more consistent comparison among studies, and so to provide highly relevant clinical results.

\section{Abbreviations}

RCR: Rotator cuff retear; RC: rotator cuff; OR: odds ratio; Cl: confidence interval; PRISMA: Preferred Reporting Items for Systematic Review and MetaAnalyses; ROM: range of motion; MRI: Magnetic Resonance Imaging; US: Ultrasound; CT: Computed Tomography; GFDI: Global Fatty Degeneration Index; PRP: Platelet-rich Plasma; PRFM: platelet-rich fibrin matrix for augmentation; LR-PRP: leukocyte-rich PRP; LP-PRP: leukocyte-poor PRP

\section{Supplementary Information}

The online version contains supplementary material available at https://doi. org/10.1186/s12891-021-04634-6.

Additional file 1. Extracted data, including first author and year of publication, study design and level of evidence, randomization groups, basic patients demographic information (i.e., age, gender), postoperative rehabilitation protocol, i.e., immobilization (Yes/No) and correspondent duration (week), beginning of passive ROM (day), active assisted ROM (mean week), full active ROM (mean weeks), strengthening exercises (mean weeks).

Additional file 2. Extracted data, surgical technique (i.e., arthroscopy, open, mini-open), preoperative tears size according to Cofield classification as small $(<1 \mathrm{~cm})$, medium $(1-3 \mathrm{~cm})$, large $(3-5 \mathrm{~cm})$, massive $(>5 \mathrm{~cm})$, RC repair (i.e., single-row, double-row, suture bridge, transosseous), diagnostic imaging tools (i.e., MRI, US, CT), number of patients undergoing 
postoperative diagnostic imaging and follow-up (mean months), number of retears either in each single randomization group than overall and correspondent retear rate, fatty infiltration of cuff muscles before surgery.

Additional file 3. Risk of bias.

\section{Acknowledgements}

The author UG Longo is a Senior Board Member for BMC Musculoskeletal Disorders.

\section{Authors' contributions}

UGL and AC conceived the study and oversaw overall direction and planning. UGL, AC, VC, and IP performed the study selection process, data extraction, and risk of bias assessment. IP performed the statistical analysis, ES verified the analytical methods and contributed to the interpretation of results. $A C$ and $A B$ wrote the manuscript in close cooperation with UGL and VD. All the authors discussed the results, read and approved the final version of the manuscript.

\section{Funding}

Not applicable.

\section{Availability of data and materials}

The datasets used and/or analysed during the current study are available from the corresponding author on reasonable request.

\section{Declarations}

\section{Ethics approval and consent to participate}

Not applicable.

\section{Consent for publication}

Not applicable.

\section{Competing interests}

The authors declare that they have no competing interests.

\section{Author details}

${ }^{1}$ Department of Orthopaedic and Trauma Surgery, Campus Bio-Medico University, Via Álvaro del Portillo, 200, Trigoria, 00128 Rome, Italy. ${ }^{2}$ Unit of Measurements and Biomedical Instrumentation, Campus Bio-Medico University, Via Álvaro del Portillo, 21, 00128 Rome, Italy.

Received: 16 October 2020 Accepted: 10 June 2021 Published online: 31 August 2021

\section{References}

1. Longo UG, Ambrogioni LR, Berton A, Candela V, Carnevale A, Schena E, et al. Physical therapy and precision rehabilitation in shoulder rotator cuff disease. Int Orthop. 2020:1-11.

2. McElvany MD, McGoldrick E, Gee AO, Neradilek MB, Matsen FA. Rotator cuff repair: published evidence on factors associated with repair integrity and clinical outcome. Am J Sports Med. 2015;43(2):491-500. https://doi.org/10.11 77/0363546514529644

3. Longo UG, Salvatore G, Rizzello G, Berton A, Ciuffreda M, Candela V, et al. The burden of rotator cuff surgery in Italy: a nationwide registry study. Arch Orthop Trauma Surg. 2017;137(2):217-24. https://doi.org/10.1007/s00402-01 6-2610-x.

4. Diebold G, Lam P, Walton J, Murrell GAC. Relationship between age and rotator Cuff Retear: a study of 1,600 consecutive rotator Cuff repairs. J Bone Joint Surg Am. 2017;99(14):1198-205. https://doi.org/10.2106/JBJS.16.00770.

5. Abtahi AM, Granger EK, Tashjian RZ. Factors affecting healing after arthroscopic rotator cuff repair. World J Orthop. 2015;6(2):211-20. https:// doi.org/10.5312/wjo.v6.i2.211.

6. Rossi LA, Chahla J, Verma NN, Millett PJ, Ranalletta M. Rotator Cuff Retears. JBJS Rev. 2020;8(1):e0039. https://doi.org/10.2106/JBJS.RVW.19.00039.

7. lijima Y, Matsuki K, Hoshika S, Ueda Y, Hamada H, Tokai M, Takahashi N, Sugaya $\mathrm{H}$, Watanabe $\mathrm{A}$. Relationship between postoperative retear and preoperative fatty degeneration in large and massive rotator cuff tears: quantitative analysis using T2 mapping. J Shoulder Elbow Surg. 2019;28(8): 1562-67. https://doi.org/10.1016/j.jse.2019.04.036.
8. Baumgarten KM, Gerlach D, Galatz LM, Teefey SA, Middleton WD, Ditsios K, et al. Cigarette smoking increases the risk for rotator cuff tears. Clin Orthop Relat Res. 2010;468(6):1534-41. https://doi.org/10.1007/s11999-009-0781-2.

9. Cho NS, Moon SC, Jeon JW, Rhee YG. The influence of diabetes mellitus on clinical and structural outcomes after arthroscopic rotator cuff repair. Am J Sports Med. 2015;43(4):991-7. https://doi.org/10.1177/0363546514565097.

10. Santiago-Torres J, Flanigan DC, Butler RB, Bishop JY. The effect of smoking on rotator cuff and glenoid labrum surgery: a systematic review. Am J Sports Med. 2015;43(3):745-51. https://doi.org/10.1177/0363546514533776.

11. Jo CH, Shin JS. Changes in appearance of fatty infiltration and muscle atrophy of rotator cuff muscles on magnetic resonance imaging after rotator cuff repair: establishing new time-zero traits. Arthroscopy. 2013;29(3): 449-58. https://doi.org/10.1016/j.arthro.2012.10.006.

12. Gartsman GM, Drake G, Edwards TB, Elkousy HA, Hammerman SM, O'Connor $D P$, et al. Ultrasound evaluation of arthroscopic full-thickness supraspinatus rotator cuff repair: single-row versus double-row suture bridge (transosseous equivalent) fixation. Results of a prospective, randomized study. J Shoulder Elb Surg. 2013;22(11):1480-7. https://doi.org/10.1016/j.jse.2013.06.020.

13. Lapner PL, Sabri E, Rakhra K, McRae S, Leiter J, Bell K, et al. A multicenter randomized controlled trial comparing single-row with double-row fixation in arthroscopic rotator cuff repair. J Bone Joint Surg Am. 2012;94(14):124957. https://doi.org/10.2106/JBJS.K.00999.

14. Nazari G, MacDermid JC, Bryant D, Dewan N, Athwal GS. Effects of arthroscopic vs. mini-open rotator cuff repair on function, pain \& range of motion. A systematic review and meta-analysis. PLoS One. 2019;14(10): e0222953.

15. Liu J, Fan L, Zhu Y, Yu H, Xu T, Li G. Comparison of clinical outcomes in allarthroscopic versus mini-open repair of rotator cuff tears: a randomized clinical trial. Medicine (Baltimore). 2017;96(11):e6322. https://doi.org/10.1097/ MD.0000000000006322.

16. Hein J, Reilly JM, Chae J, Maerz T, Anderson K. Retear rates after arthroscopic single-row, double-row, and suture bridge rotator Cuff repair at a minimum of 1 year of imaging follow-up: a systematic review. Arthroscopy : the journal of arthroscopic \& related surgery : official publication of the Arthroscopy Association of North America and the International Arthroscopy Association. 2015;31(11):2274-81. https://doi.org/10.1016/j.a rthro.2015.06.004

17. Muller AM, Flury M, Alsayed HN, Audige L. Influence of patient and diagnostic parameters on reported retear rates after arthroscopic rotator cuff repair. Knee Surg Sports Traumatol Arthrosc. 2017;25(7):2089-99. https://doi.org/10.1007/s00167-017-4481-2.

18. Houck DA, Kraeutler MJ, Schuette HB, McCarty EC, Bravman JT. Early versus delayed motion after rotator Cuff repair: a systematic review of overlapping Meta-analyses. Am J Sports Med. 2017;45(12):2911-5. https://doi.org/10.11 77/0363546517692543.

19. Kim YS, Lee HJ, Park I, Im JH, Park KS, Lee SB. Are delayed operations effective for patients with rotator cuff tears and concomitant stiffness? An analysis of immediate versus delayed surgery on outcomes. Arthroscopy. 2015;31(2):197-204. https://doi.org/10.1016/j.arthro.2014.08.014.

20. Barth J, Andrieu K, Fotiadis E, Hannink G, Barthelemy R, Saffarini M. Critical period and risk factors for retear following arthroscopic repair of the rotator cuff. Knee Surg Sports Traumatol Arthrosc. 2017;25(7):2196-204. https://doi. org/10.1007/s00167-016-4276-X

21. lannotti JP, Deutsch A, Green A, Rudicel S, Christensen J, Marraffino S, et al. Time to failure after rotator cuff repair: a prospective imaging study. J Bone Joint Surg Am. 2013;95(11):965-71. https://doi.org/10.2106/JBJS.L.00708.

22. Longo UG, Rizzello G, Petrillo S, Loppini M, Maffulli N, Denaro V. Conservative Rehabilitation Provides Superior Clinical Results Compared to Early Aggressive Rehabilitation for Rotator Cuff Repair: A Retrospective Comparative Study. Medicina (Kaunas). 2019;55(8):402. https://doi.org/10.33 90/medicina55080402.

23. Riboh JC, Garrigues GE. Early passive motion versus immobilization after arthroscopic rotator cuff repair. Arthroscopy. 2014;30(8):997-1005. https:// doi.org/10.1016/j.arthro.2014.03.012.

24. Li S, Sun H, Luo X, Wang K, Wu G, Zhou J, et al. The clinical effect of rehabilitation following arthroscopic rotator cuff repair: a meta-analysis of early versus delayed passive motion. Medicine (Baltimore). 2018;97(2):e9625. https://doi.org/10.1097/MD.0000000000009625.

25. Le BT, Wu XL, Lam PH, Murrell GA. Factors predicting rotator cuff retears: an analysis of 1000 consecutive rotator cuff repairs. Am J Sports Med. 2014; 42(5):1134-42. https://doi.org/10.1177/0363546514525336. 
26. Liberati A, Altman DG, Tetzlaff J, Mulrow C, Gøtzsche PC, loannidis JP, et al. The PRISMA statement for reporting systematic reviews and meta-analyses of studies that evaluate healthcare interventions: explanation and elaboration. BMJ. 2009;339(jul21 1):b2700. https://doi.org/10.1136/bmj.b2700.

27. Marx RG, Wilson SM, Swiontkowski MF. Updating the assignment of levels of evidence. J Bone Joint Surg Am. 2015;97(1):1-2. https://doi.org/10.2106/ JBJS.N.01112.

28. Koh KH, Kang KC, Lim TK, Shon MS, Yoo JC. Prospective randomized clinical trial of single- versus double-row suture anchor repair in 2- to 4-cm rotator cuff tears: clinical and magnetic resonance imaging results. Arthroscopy. 2011;27(4):453-62. https://doi.org/10.1016/j.arthro.2010.11.059.

29. Cofield RH. Rotator cuff disease of the shoulder. J Bone Joint Surg Am 1985;67(6):974-9. https://doi.org/10.2106/00004623-198567060-00024.

30. van Tulder M, Furlan A, Bombardier C, Bouter L. Group EBotCCBR: updated method guidelines for systematic reviews in the cochrane collaboration back review group. Spine (Phila Pa 1976). 2003;28(12):1290-9. https://doi. org/10.1097/01.BRS.0000065484.95996.AF.

31. Cumpston M, Li T, Page MJ, Chandler J, Welch VA, Higgins JP, et al. Updated guidance for trusted systematic reviews: a new edition of the Cochrane handbook for systematic reviews of interventions. Cochrane Database Syst Rev. 2019;10:ED000142.

32. Shamseer L, Moher D, Clarke M, Ghersi D, Liberati A, Petticrew M, et al. Preferred reporting items for systematic review and meta-analysis protocols (PRISMA-P) 2015: elaboration and explanation. BMJ. 2015;350:g7647.

33. Burks RT, Crim J, Brown N, Fink B, Greis PE. A prospective randomized clinical trial comparing arthroscopic single- and double-row rotator cuff repair: magnetic resonance imaging and early clinical evaluation. Am J Sports Med. 2009;37(4):674-82. https://doi.org/10.1177/0363546508328115.

34. Carbonel I, Martinez AA, Calvo A, Ripalda J, Herrera A. Single-row versus double-row arthroscopic repair in the treatment of rotator cuff tears: a prospective randomized clinical study. Int Orthop. 2012;36(9):1877-83. https://doi.org/10.1007/s00264-012-1559-9.

35. Castricini R, Longo UG, De Benedetto M, Panfoli N, Pirani P, Zini R, et al. Platelet-rich plasma augmentation for arthroscopic rotator cuff repair: a randomized controlled trial. Am J Sports Med. 2011;39(2):258-65. https://doi. org/10.1177/0363546510390780.

36. D'Ambrosi R, Palumbo F, Paronzini A, Ragone V, Facchini RM. Platelet-rich plasma supplementation in arthroscopic repair of full-thickness rotator cuff tears: a randomized clinical trial. Musculoskelet Surg. 2016;100(Suppl 1):2532. https://doi.org/10.1007/s12306-016-0415-2.

37. Ebert JR, Wang A, Smith A, Nairn R, Breidahl W, Zheng MH, et al. A midterm evaluation of postoperative platelet-rich plasma injections on arthroscopic supraspinatus repair: a randomized controlled trial. Am J Sports Med. 2017; 45(13):2965-74. https://doi.org/10.1177/0363546517719048.

38. Flury M, Rickenbacher D, Schwyzer HK, Jung C, Schneider MM, Stahnke K, et al. Does pure platelet-rich plasma affect postoperative clinical outcomes after arthroscopic rotator Cuff repair? A randomized controlled trial. Am J Sports Med. 2016;44(8):2136-46. https://doi.org/10.1177/0363546516645518.

39. Franceschi F, Ruzzini L, Longo UG, Martina FM, Zobel BB, Maffulli N, et al. Equivalent clinical results of arthroscopic single-row and double-row suture anchor repair for rotator cuff tears: a randomized controlled trial. Am J Sports Med. 2007;35(8):1254-60. https://doi.org/10.1177/0363546507302218.

40. Franceschi F, Papalia R, Franceschetti E, Palumbo A, Del Buono A, Paciotti M, et al. Double-row repair lowers the Retear risk after accelerated rehabilitation. Am J Sports Med. 2016;44(4):948-56. https://doi.org/10.1177/ 0363546515623031.

41. Gumina S, Passaretti D, Gurzi MD, Candela V. Arginine L-alpha-ketoglutarate, methylsulfonylmethane, hydrolyzed type I collagen and bromelain in rotator cuff tear repair: a prospective randomized study. Curr Med Res Opin. 2012;28(11):1767-74. https://doi.org/10.1185/03007995.2012.737772.

42. Gumina S, Campagna V, Ferrazza G, Giannicola G, Fratalocchi F, Milani A, et al. Use of platelet-leukocyte membrane in arthroscopic repair of large rotator cuff tears: a prospective randomized study. J Bone Joint Surg Am. 2012;94(15):1345-52. https://doi.org/10.2106/JBJS.K.00394

43. Jacquot A, Dezaly C, Goetzmann T, Roche O, Sirveaux F, Mole D. Is rotator cuff repair appropriate in patients older than 60 years of age? Prospective, randomised trial in 103 patients with a mean four-year follow-up. Orthop Traumatol Surg Res. 2014;100(6 Suppl):S333-8. https://doi.org/10.1016/j. otsr.2014.04.004

44. Jo CH, Shin JS, Lee YG, Shin WH, Kim H, Lee SY, et al. Platelet-rich plasma for arthroscopic repair of large to massive rotator cuff tears: a randomized, single-blind, parallel-group trial. Am J Sports Med. 2013;41(10):2240-8. https://doi.org/10.1177/0363546513497925.

45. Kim YS, Lee HJ, Jin HK, Kim SE, Lee JW. Conventional En masse repair versus separate double-layer double-row repair for the treatment of delaminated rotator Cuff tears. Am J Sports Med. 2016;44(5):1146-52. https://doi.org/1 $0.1177 / 0363546516628869$.

46. Ma HL, Chiang ER, Wu HT, Hung SC, Wang ST, Liu CL, et al. Clinical outcome and imaging of arthroscopic single-row and double-row rotator cuff repair: a prospective randomized trial. Arthroscopy. 2012;28(1):16-24. https://doi. org/10.1016/j.arthro.2011.07.003.

47. Malavolta EA, Gracitelli MEC, Assunção JH, Ferreira Neto AA, BordaloRodrigues M, de Camargo OP. Clinical and structural evaluations of rotator Cuff repair with and without added platelet-rich plasma at 5-year follow-up: a prospective randomized study. Am J Sports Med. 2018;46(13):3134-41. https://doi.org/10.1177/0363546518795895.

48. Pandey V, Bandi A, Madi S, Agarwal L, Acharya KK, Maddukuri S, et al. Does application of moderately concentrated platelet-rich plasma improve clinical and structural outcome after arthroscopic repair of medium-sized to large rotator cuff tear? A randomized controlled trial. J Shoulder Elb Surg. 2016; 25(8):1312-22. https://doi.org/10.1016/j.jse.2016.01.036.

49. Randelli P, Stoppani CA, Zaolino C, Menon A, Randelli F, Cabitza P. Advantages of arthroscopic rotator Cuff repair with a Transosseous suture technique: a prospective randomized controlled trial. Am J Sports Med. 2017;45(9):2000-9. https://doi.org/10.1177/0363546517695789.

50. Randelli P, Arrigoni P, Ragone V, Aliprandi A, Cabitza P. Platelet rich plasma in arthroscopic rotator cuff repair: a prospective RCT study, 2-year follow-up. J Shoulder Elbow Surg / Am Shoulder Elbow Surg [et al]. 2011;20(4):518-28. https://doi.org/10.1016/j.jse.2011.02.008.

51. Rodeo SA, Delos D, Williams RJ, Adler RS, Pearle A, Warren RF. The effect of platelet-rich fibrin matrix on rotator cuff tendon healing: a prospective, randomized clinical study. Am J Sports Med. 2012;40(6):1234-41. https://doi. org/10.1177/0363546512442924.

52. Ruiz-Moneo P, Molano-Munoz J, Prieto E, Algorta J. Plasma rich in growth factors in arthroscopic rotator cuff repair: a randomized, double-blind, controlled clinical trial. Arthroscopy : the journal of arthroscopic \& related surgery : official publication of the Arthroscopy Association of North America and the International Arthroscopy Association. 2013;29(1):2-9. https://doi.org/10.1016/j.arthro.2012.08.014.

53. Shin SJ, Oh JH, Chung SW, Song MH. The efficacy of acromioplasty in the arthroscopic repair of small- to medium-sized rotator cuff tears without acromial spur: prospective comparative study. Arthroscopy. 2012;28(5):62835. https://doi.org/10.1016/j.arthro.2011.10.016.

54. Walsh MR, Nelson BJ, Braman JP, Yonke B, Obermeier M, Raja A, et al. Platelet-rich plasma in fibrin matrix to augment rotator cuff repair: a prospective, single-blinded, randomized study with 2-year follow-up. J Shoulder Elb Surg. 2018;27(9):1553-63. https://doi.org/10.1016/j.jse.2018.05. 003.

55. Wang A, McCann P, Colliver J, Koh E, Ackland T, Joss B, et al. Do postoperative platelet-rich plasma injections accelerate early tendon healing and functional recovery after arthroscopic supraspinatus repair? A randomized controlled trial. Am J Sports Med. 2015;43(6):1430-7. https://doi. org/10.1177/0363546515572602.

56. Weber SC, Kauffman JI, Parise C, Weber SJ, Katz SD. Platelet-rich fibrin matrix in the management of arthroscopic repair of the rotator cuff: a prospective, randomized, double-blinded study. Am J Sports Med. 2013:41(2):263-70. https://doi.org/10.1177/0363546512467621.

57. Zumstein MA, Rumian A, Thélu C, Lesbats V, O'Shea K, Schaer M, et al. SECEC research Grant 2008 Il: use of platelet- and leucocyte-rich fibrin (LPRF) does not affect late rotator cuff tendon healing: a prospective randomized controlled study. J Shoulder Elb Surg. 2016;25(1):2-11. https:// doi.org/10.1016/j.jse.2015.09.018.

58. Barber FA, Burns JP, Deutsch A, Labbe MR, Litchfield RB. A prospective, randomized evaluation of acellular human dermal matrix augmentation for arthroscopic rotator cuff repair. Arthroscopy. 2012;28(1):8-15. https://doi. org/10.1016/j.arthro.2011.06.038.

59. Cai YZ, Zhang C, Jin RL, Shen T, Gu PC, Lin XJ, et al. Arthroscopic rotator Cuff repair with graft augmentation of 3-dimensional biological collagen for moderate to large tears: a randomized controlled study. Am J Sports Med. 2018;46(6):1424-31. https://doi.org/10.1177/0363546518756978.

60. Greiner S, Ide J, Van Noort A, Mochizuki Y, Ochi H, Marraffino S, et al. Local rhBMP-12 on an absorbable collagen sponge as an adjuvant therapy for 
rotator Cuff repair - a phase 1, randomized, standard of care control, multicenter study: safety and feasibility. Am J Sports Med. 2015;43(8):19942004. https://doi.org/10.1177/0363546515584756.

61. Lamas JR, García-Fernández C, Tornero-Esteban P, Lópiz Y, RodriguezRodriguez $L$, Ortega $L$, et al. Adverse effects of xenogenic scaffolding in the context of a randomized double-blind placebo-controlled study for repairing full-thickness rotator cuff tears. Trials. 2019;20(1):387. https://doi. org/10.1186/s13063-019-3504-3.

62. lannotti JP, Codsi MJ, Kwon YW, Derwin K, Ciccone J, Brems JJ. Porcine small intestine submucosa augmentation of surgical repair of chronic twotendon rotator cuff tears. A randomized, controlled trial. J Bone Joint Surg Am. 2006;88(6):1238-44. https://doi.org/10.2106/00004623-200606000-00010.

63. Ide J, Mochizuki Y, van Noort A, Ochi H, Sridharan S, Itoi E, et al. Local rhBMP-12 on an absorbable collagen sponge as an adjuvant therapy for rotator Cuff repair-a phase 1, randomized, standard of care control, multicenter study: part 2-a pilot study of functional recovery and structura outcomes. Orthop J Sports Med. 2017;5(9):2325967117726740. https://doi. org/10.1177/2325967117726740.

64. Cuff DJ, Pupello DR. Prospective randomized study of arthroscopic rotator cuff repair using an early versus delayed postoperative physical therapy protocol. J Shoulder Elbow Surg / Am Shoulder Elbow Surg [et al]. 2012; 21(11):1450-5. https://doi.org/10.1016/j.jse.2012.01.025.

65. Jenssen KK, Lundgreen K, Madsen JE, Kvakestad R, Pripp AH, Dimmen S. No functional difference between three and six weeks of immobilization after arthroscopic rotator Cuff repair: a prospective randomized controlled noninferiority trial. Arthroscopy. 2018;34(10):2765-74. https://doi.org/10.1016/j.a rthro.2018.05.036.

66. Kim YS, Chung SW, Kim JY, Ok JH, Park I, Oh JH. Is early passive motion exercise necessary after arthroscopic rotator cuff repair? Am J Sports Med. 2012;40(4):815-21. https://doi.org/10.1177/0363546511434287.

67. Koh KH, Lim TK, Shon MS, Park YE, Lee SW, Yoo JC. Effect of immobilization without passive exercise after rotator cuff repair: randomized clinical trial comparing four and eight weeks of immobilization. J Bone Joint Surg Am. 2014;96(6):e44. https://doi.org/10.2106/JBJS.L.01741.

68. Lee BG, Cho NS, Rhee YG. Effect of two rehabilitation protocols on range of motion and healing rates after arthroscopic rotator cuff repair: aggressive versus limited early passive exercises. Arthroscopy : the journal of arthroscopic \& related surgery : official publication of the Arthroscopy Association of North America and the International Arthroscopy Association. 2012;28(1):34-42. https://doi.org/10.1016/j.arthro.2011.07.012.

69. Mazzocca AD, Arciero RA, Shea KP, Apostolakos JM, Solovyova O, Gomlinski $G$, et al. The effect of early range of motion on quality of life, clinical outcome, and repair integrity after arthroscopic rotator Cuff repair Arthroscopy. 2017;33(6):1138-48. https://doi.org/10.1016/j.arthro.2016.10.017.

70. Sheps DM, Silveira A, Beaupre L, Styles-Tripp F, Balyk R, Lalani A, et al. Early Active Motion Versus Sling Immobilization After Arthroscopic Rotator Cuff Repair: A Randomized Controlled Trial. Arthroscopy. 2019;35(3):749-760.e742.

71. Lee HJ, Jeong JY, Kim CK, Kim YS. Surgical treatment of lesions of the long head of the biceps brachii tendon with rotator cuff tear: a prospective randomized clinical trial comparing the clinical results of tenotomy and tenodesis. J Shoulder Elb Surg. 2016;25(7):1107-14. https://doi.org/10.1016/j. jse.2016.02.006.

72. Carr A, Cooper C, Campbell MK, Rees J, Moser J, Beard DJ, et al. Effectiveness of open and arthroscopic rotator cuff repair (UKUFF): a randomised controlled trial. Bone Joint J. 2017;99-B(1):107-15. https://doi. org/10.1302/0301-620X.99B1.BJJ-2016-0424.R1.

73. Bidwai AS, Birch A, Temperley D, Odak S, Walton MJ, Haines JF, et al. Medium- to long-term results of a randomized controlled trial to assess the efficacy of arthoscopic-subacromial decompression versus mini-open repair for the treatment of medium-sized rotator cuff tears. Shoulder Elbow. 2016; 8(2):101-5. https://doi.org/10.1177/1758573215620571.

74. Nam JH, Park S, Lee HR, Kim SH. Outcomes after limited or extensive Bursectomy during rotator Cuff repair: randomized controlled trial. Arthroscopy. 2018;34(12):3167-74. https://doi.org/10.1016/j.arthro.2018.06.056.

75. Boehm TD, Werner A, Radtke S, Mueller T, Kirschner S, Gohlke F. The effect of suture materials and techniques on the outcome of repair of the rotator cuff: a prospective, randomised study. J Bone Joint Surg Br. 2005;87(6):81923.

76. Osti L, Del Buono A, Maffulli N. Microfractures at the rotator cuff footprint: a randomised controlled study. Int Orthop. 2013;37(11):2165-71. https://doi. org/10.1007/s00264-013-1952-z
77. Tirefort J, Schwitzguebel AJ, Collin P, Nowak A, Plomb-Holmes C, Ladermann A. Postoperative mobilization after superior rotator Cuff repair: sling versus no sling: a randomized prospective study. J Bone Joint Surg Am. 2019;101(6):494-503. https://doi.org/10.2106/JBJS.18.00773.

78. Zhang Z, Wang Y, Sun J. The effect of platelet-rich plasma on arthroscopic double-row rotator cuff repair: a clinical study with 12-month follow-up. Acta Orthop Traumatol Turc. 2016;50(2):191-7. https://doi.org/10.3944/A OTT.2015.15.0113.

79. van der Zwaal P, Thomassen BJ, Nieuwenhuijse MJ, Lindenburg R, Swen JW, van Arkel ER. Clinical outcome in all-arthroscopic versus mini-open rotator cuff repair in small to medium-sized tears: a randomized controlled trial in 100 patients with 1-year follow-up. Arthroscopy. 2013;29(2):266-73. https:// doi.org/10.1016/j.arthro.2012.08.022

80. Rhee YG, Cho NS, Parke CS. Arthroscopic rotator cuff repair using modified Mason-Allen medial row stitch: knotless versus knot-tying suture bridge technique. Am J Sports Med. 2012;40(11):2440-7. https://doi.org/10.1177/03 63546512459170

81. Keener JD, Galatz LM, Stobbs-Cucchi G, Patton R, Yamaguchi K. Rehabilitation following arthroscopic rotator cuff repair: a prospective randomized trial of immobilization compared with early motion. J Bone Joint Surg Am. 2014:96(1):11-9. https://doi.org/10.2106/JBJS.M.00034.

82. Antuna S, Barco R, Martinez Diez JM, Sanchez Marquez JM. Platelet-rich fibrin in arthroscopic repair of massive rotator cuff tears: a prospective randomized pilot clinical trial. Acta Orthop Belg. 2013;79(1):25-30.

83. Kim JY, Lee JS, Park CW. Extracorporeal shock wave therapy is not useful after arthroscopic rotator cuff repair. Knee Surg Sports Traumatol Arthrosc. 2012;20(12):2567-72. https://doi.org/10.1007/s00167-012-1923-8.

84. De Roo PJ, Muermans S, Maroy M, Linden P, Van den Daelen L. Passive mobilization after arthroscopic rotator cuff repair is not detrimental in the early postoperative period. Acta Orthop Belg. 2015;81(3):485-92.

85. Lambers Heerspink FO, van Raay JJAM, Koorevaar RCT, van Eerden PJM, Westerbeek RE, Van't Riet E, et al. Comparing surgical repair with conservative treatment for degenerative rotator cuff tears: a randomized controlled trial. J Shoulder Elb Surg. 2015;24(8):1274-81. https://doi.org/10.1 016/j.jse.2015.05.040

86. Moosmayer S, Lund G, Seljom US, Haldorsen B, Svege IC, Hennig T, et al. At a 10-year follow-up, tendon repair is superior to physiotherapy in the treatment of small and medium-sized rotator Cuff tears. J Bone Joint Surg Am. 2019;101(12):1050-60. https://doi.org/10.2106/JBJS.18.01373.

87. Avanzi P, Giudici LD, Capone A, Cardoni G, Lunardi G, Foti G, et al. Prospective randomized controlled trial for patch augmentation in rotator cuff repair: 24-month outcomes. J Shoulder Elb Surg. 2019;28(10):1918-27. https://doi.org/10.1016/j.jse.2019.05.043.

88. Goutallier D, Postel JM, Gleyze P, Leguilloux P, Van Driessche S. Influence of cuff muscle fatty degeneration on anatomic and functional outcomes after simple suture of full-thickness tears. J Shoulder Elb Surg. 2003;12(6):550-4. https://doi.org/10.1016/S1058-2746(03)00211-8.

89. MOON Shoulder Post-operative Rotator Cuff Repair Protocol - Therapist Instructions, https://moonshoulder.com/wpcontent/uploads/2020/04/ Immediate-Post-operative-Rotator-Cuff-Repair-Protocol-TherapistInstructions.pdf.

90. Altintas B, Anderson NL, Pitta R, Buckley PS, Bhatia S, Provencher MT, et al. Repair of rotator Cuff tears in the elderly: does it make sense? A systematic review. Am J Sports Med. 2020;48(3):744-53. https://doi.org/10.1177/036354 6519834574.

91. Saccomanno MF, Sircana G, Cazzato G, Donati F, Randelli P, Milano G. Prognostic factors influencing the outcome of rotator cuff repair: a systematic review. Knee Surg Sports Traumatol Arthrosc. 2016;24(12):380919. https://doi.org/10.1007/s00167-015-3700-y.

92. Khair MM, Lehman J, Tsouris N, Gulotta LV. A systematic review of preoperative fatty infiltration and rotator Cuff outcomes. HSS J. 2016;12(2): 170-6. https://doi.org/10.1007/s11420-015-9465-5.

93. Chung SW, Kim JY, Kim MH, Kim SH, Oh JH. Arthroscopic repair of massive rotator cuff tears: outcome and analysis of factors associated with healing failure or poor postoperative function. Am J Sports Med. 2013;41(7):167483. https://doi.org/10.1177/0363546513485719.

94. Longo UG, Berton A, Risi Ambrogioni L, Lo Presti D, Carnevale A, Candela V, Stelitano G, Schena E, Nazarian A, Denaro V. Cost-Effectiveness of Supervised versus Unsupervised Rehabilitation for Rotator-Cuff Repair: Systematic Review and Meta-Analysis. Int J Environ Res Public Health. 2020; 17(8):2852. https://doi.org/10.3390/ijerph17082852. 
95. Jung C, Tepohl L, Tholen R, Beitzel K, Buchmann S, Gottfried T, et al. Rehabilitation following rotator cuff repair: A work of the Commission Rehabilitation of the German Society of Shoulder and Elbow Surgery e.V. (DVSE) in collaboration with the German Association for Physiotherapy (ZVK) e.V., the Association Physical Therapy, Association for Physical Professions (VPT) e.V. and the Section Rehabilitation-Physical Therapy of the German Society for Orthopaedics and Trauma e.V. (DGOU). Obere Extrem. 2018;13(1):45-61.

96. Sheps DM, Silveira A, Beaupre L, Styles-Tripp F, Balyk R, Lalani A, et al. Early Active Motion Versus Sling Immobilization After Arthroscopic Rotator Cuff Repair: A Randomized Controlled Trial. Arthroscopy. 2019;35(3):749-760. e742.

97. Carnevale A, Longo UG, Schena E, Massaroni C, Lo Presti D, Berton A, et al. Wearable systems for shoulder kinematics assessment: a systematic review. BMC Musculoskelet Disord. 2019;20(1):546. https://doi.org/10.1186/s12891-01 9-2930-4.

98. Kang L, Henn RF, Tashijan RZ, Green A. Early outcome of arthroscopic rotator cuff repair: a matched comparison with mini-open rotator cuff repair. Arthroscopy. 2007;23(6):573-82.

99. Pearsall AW, Ibrahim KA, Madanagopal SG. The results of arthroscopic versus mini-open repair for rotator cuff tears at mid-term follow-up. J Orthop Surg Res. 2007;2(1):24. https://doi.org/10.1186/1749-799X-2-24

100. Rossi LA, Rodeo SA, Chahla J, Ranalletta M. Current concepts in rotator Cuff repair techniques: biomechanical, functional, and structural outcomes. Orthop J Sports Med. 2019;7(9):2325967119868674. https://doi.org/10.11 77/2325967119868674.

101. Yang J Jr, Robbins M, Reilly J, Maerz T, Anderson K. The clinical effect of a rotator Cuff Retear: a Meta-analysis of arthroscopic single-row and doublerow repairs. Am J Sports Med. 2017;45(3):733-41. https://doi.org/10.1177/03 63546516652900.

102. Lin MT, Wei KC, Wu CH. Effectiveness of Platelet-Rich Plasma Injection in Rotator Cuff Tendinopathy: A Systematic Review and Meta-Analysis of Randomized Controlled Trials. Diagnostics (Basel). 2020;10(4):189. https://doi. org/10.3390/diagnostics10040189.

\section{Publisher's Note}

Springer Nature remains neutral with regard to jurisdictional claims in published maps and institutional affiliations.

Ready to submit your research? Choose BMC and benefit from:

- fast, convenient online submission

- thorough peer review by experienced researchers in your field

- rapid publication on acceptance

- support for research data, including large and complex data types

- gold Open Access which fosters wider collaboration and increased citations

- maximum visibility for your research: over $100 \mathrm{M}$ website views per year

At $\mathrm{BMC}$, research is always in progress.

Learn more biomedcentral.com/submissions 